\title{
COMPOSITE FINITE ELEMENTS FOR ELLIPTIC INTERFACE PROBLEMS
}

\author{
DANIEL PETERSEIM
}

\begin{abstract}
A Composite Finite Element method approximates linear elliptic boundary value problems with discontinuous diffusion coefficient at possibly high contrast. The discontinuity appears at some interface that is not necessarily resolved by the underlying finite element mesh. The method is nonconforming in the sense that shape functions preserve continuity across the interface in only an approximate way. However, the method allows balancing this non-conformity error and the error of the best approximation in such a way that the total discretization error (in energy norm) decreases linear with regard to the mesh size and independent of contrast.
\end{abstract}

\section{INTRODUCTION}

This research article considers the design of a Composite Finite Element (CFE) method for Dirichlet problems with discontinuous coefficients across an interface. The CFE method is a classical two-scale approach: The degrees of freedom are related to a possibly coarse mesh, whereas the shape of the ansatz functions is defined on a finer subgrid. In other words, finite element shape functions on a coarse scale are composite by shape functions from some finer scale.

In previous CFEs [18, 19,22, for the treatment of essential boundary conditions on unfitted meshes (with respect to the boundary of the domain), the adaptation of shape was done in such a way that the prescribed boundary condition was fulfilled in an approximate way. Now, in the context of interface problems, finite element shape functions are adapted on a submesh such that the continuity across the interface is preserved in an approximate way. The new CFE approach has three main advantages:

(1) The definition of basis functions is explicit, i.e., no local problems have to be solved.

(2) The coarse mesh does not need to be aligned with the interface, whereas this is necessary for classical finite element methods (see [14]) to converge at an optimal rate. Moreover, the definition of the CFE method does not put any condition on the intersection of mesh cells and the interface.

(3) If the given data (domain, interface, right-hand side, etc.) allow for a (piecewise) smooth solution, the asymptotic order of convergence of the

Received by the editor October 25, 2010 and, in revised form, January 16, 2012 and February $16,2013$.

2010 Mathematics Subject Classification. Primary 65N30, 65N12, 35R05, 80M10.

The present paper is a full version of an extended abstract presented at the 81st Annual Meeting of the International Association of Applied Mathematics and Mechanics, Karlsruhe (Germany), 2010. The work was partially supported by the DFG Research Center MATHEON Berlin through project C33. 
underlying discretization is preserved on coarse meshes which do not resolve the interface.

Alternative approaches in the literature can be found, for instance, in [24], where another CFE method is introduced, in [1, 9, where the interface condition is imposed weakly via penalization, or in [4, where special basis functions are computed by solving local problems on submeshes.

The present CFE method may be useful for problems with evolving interfaces. Because of evolution, the interface cannot be well represented by edges or faces of a stationary mesh. In classical finite element methods, an adaptation of the mesh to the interface at every time step is required. This adaptation of the mesh in time is considered to be too costly, especially in three space dimensions. The new CFE approach allows the computing of the evolution in time on a fixed (possibly coarse) mesh. It is sufficient to adapt the shape of the ansatz functions (slightly, close to the interface) in time. As we will see later, the cost for this shape adaptation is small when compared with the overall cost of updating the solution on the fixed coarse mesh.

Note finally that our method is designed to efficiently treat the singularity caused by the jump of the diffusion coefficient at the interface. Since the method does not add any degrees of freedom to the coarse finite element space to resolve the interface, it cannot be expected to resolve any singular behavior caused, e.g., by a kink in the interface. The treatment of such singularities has to be organized on top by classical techniques, e.g., by enrichment of the finite element space by certain singular functions or by mesh adaptivity. In the context of adaptivity, CFEs offer a coarse grid approximation that may serve as the initial guess for an a-posterioridriven adaptive refinement process. They allow the adaptivity toward singularities to start long before the interface is resolved by the underlying finite element mesh.

Notation. In what follows, dist $(\cdot, \cdot)$ denotes the Euclidean distance in $\mathbb{R}^{2}$. We use the same notation for the distance between non-empty subsets $A, B \subset \mathbb{R}^{2}$, $\operatorname{dist}(A, B):=\inf _{x \in A, y \in B} \operatorname{dist}(x, y)$.

The measure $|\cdot|$ is also context-sensitive and refers to the volume of a set relative to its dimension, i.e., $|\cdot|$ denotes the length of a curve, or the area of a domain.

Given some bounded domain $\Omega$, standard notation for (fractional) Sobolev spaces $W_{p}^{m}(\Omega), m \geq 0, p \in \mathbb{N} \cup\{\infty\}$, and their corresponding norms $\|\cdot\|_{W_{p}^{m}(\Omega)}$ and seminorms $|\cdot|_{W_{p}^{m}(\Omega)}$ is used; $H^{m}(\Omega)$ abbreviates $W_{2}^{m}(\Omega)(m \in \mathbb{N})$ and $L^{p}(\Omega)$ abbreviates $W_{p}^{0}(\Omega)$. Given two disjoint bounded Lipschitz domains $\Omega_{1}$ and $\Omega_{2}$, the space $H^{m}\left(\Omega_{1} \cup \Omega_{2}\right)$ denotes the space of all functions $u \in L^{2}\left(\Omega_{1} \cup \Omega_{2}\right)$ with $\left.u\right|_{\Omega_{1}} \in H^{m}\left(\Omega_{1}\right)$ and $\left.u\right|_{\Omega_{2}} \in H^{m}\left(\Omega_{2}\right)$. The dual space of a Hilbert space $V$ is indicated by $V^{*}$. The space of $\mathbb{R}$-valued continuous functions on a set $\Omega$ is denoted by $C^{0}(\Omega)$.

\section{Composite finite element discretization of A MODEL POISSON PROBLEM}

2.1. Model problem. Consider Poisson's equation $-\operatorname{div}(a \nabla u)=f$ in an open, bounded, polyhedral domain $\Omega \subset \mathbb{R}^{d}, d \in\{2,3\}$, with homogeneous Dirichlet boundary conditions on $\partial \Omega$. The scalar coefficient $a$ (permeability or conductivity) jumps across an interface $\Gamma:=\bar{\Omega}_{1} \cap \bar{\Omega}_{2}$ that separates two disjoint, open Lipschitz subdomains $\Omega_{1}, \Omega_{2} \subset \Omega, \bar{\Omega}=\bar{\Omega}_{1} \cup \bar{\Omega}_{2}$. The corresponding variational problem 
reads: Find $u^{\star} \in H_{0}^{1}(\Omega)$ such that

$$
\int_{\Omega} a \nabla u^{\star} \cdot \nabla v \mathrm{~d} x=\int_{\Omega} f v \mathrm{~d} x \quad \text { for all } v \in H_{0}^{1}(\Omega) .
$$

For simplicity, the coefficient $a: \Omega \rightarrow \mathbb{R}_{>0}$ is chosen piecewise constant,

$$
a(x)= \begin{cases}1 & \text { if } x \in \Omega_{1}, \\ a_{\text {cont }}>1 & \text { if } x \in \Omega_{2} .\end{cases}
$$

The parameter $a_{\text {cont }}$ represents the contrast which is supposed to be large in practical applications, e.g., in the modeling of heat transfer in composite materials.

The bounded bilinear form $\mathfrak{a}: H_{0}^{1}(\Omega) \times H_{0}^{1}(\Omega) \rightarrow \mathbb{R}$ given by

$$
\mathfrak{a}(u, v):=\int_{\Omega} a \nabla u \cdot \nabla v \mathrm{~d} x=\int_{\Omega_{1}} \nabla u \cdot \nabla v \mathrm{~d} x+a_{\text {cont }} \int_{\Omega_{2}} \nabla u \cdot \nabla v \mathrm{~d} x
$$

for $u, v \in H_{0}^{1}(\Omega)$ induces the norm $\||\cdot|\|:=\|\sqrt{\bar{a}} \nabla \cdot\|_{L^{2}(\Omega)}$ in $H_{0}^{1}(\Omega)$, the so-called energy norm. Hence, problem (2.1) has a unique solution for all $f \in H^{-1}(\Omega):=$ $\left(H_{0}^{1}(\Omega)\right)^{*}$.

Usually, some finite-dimensional subspace $V_{h} \subset H_{0}^{1}(\Omega)$ based on piecewise polynomials replaces $H_{0}^{1}(\Omega)$ in a finite element discretization of (2.1). However, if the underlying finite element mesh is not aligned with the interface, this ansatz suffers from the lack of regularity of the solution at the interface; the solution is continuous across, but its gradient may jump.

In this paper, this issue shall be fixed by considering a discrete space $V_{h}$ that violates conformity, $V_{h} \not \subset H_{0}^{1}(\Omega)$. We consider shape functions that are conforming with respect to each of the subdomains but possibly discontinuous across the interface, i.e.,

$$
V_{h} \subset H_{0}^{1}\left(\Omega_{1} \cup \Omega_{2}\right):=\left\{u \in H^{1}\left(\Omega_{1} \cup \Omega_{2}\right):\left.u\right|_{\partial \Omega}=0\right\} .
$$

Because of the lack of Galerkin orthogonality, the discretization error of a corresponding method is not necessarily proportional to the error of the best approximation of the solution. The discretization error is bounded by the sum of the best approximation error and the error related to the violation of conformity as in (3.1). The aim of this paper is to construct a non-conforming discrete space $V_{h}$ (based on piecewise affine ansatz functions) such that a balance is achieved between the errors due to non-conformity and errors due to best approximation. This balance yields linear convergence of the corresponding method with respect to the mesh size parameter $h$ without resolving the interface by degrees of freedom.

2.2. Construction of the finite element space. The construction in Subsections 2.2.1 2.2.3 below follows the methodology of CFEs [8].

2.2.1. Triangulations. Let $\mathcal{T}$ be some regular subdivision of $\bar{\Omega}$ into closed nonempty simplices (or triangulation for short) according to Ciarlet [3, 5]. Two nondisjoint distinct simplices in $\mathcal{T}$ share either a common face $(d=3)$, a common edge, or a common vertex. By $V(T)$ we denote the set of vertices (corners) of a simplex $T \in \mathcal{T}$. The union of vertices in a (sub)triangulation $\mathcal{T}$ is denoted by $V(\mathcal{T}):=\bigcup_{T \in \mathcal{T}} V(T)$. The $\mathcal{T}$-piecewise mesh width function $h: \bar{\Omega} \rightarrow \mathbb{R}_{>0}$ is given by

$$
h(x):=\max _{T \in \mathcal{T}: x \in T} \operatorname{diam}(T) .
$$


Note that the coarse triangulation $\mathcal{T}$ does not necessarily match the interface $\Gamma$, i.e., $\Gamma$ is not the union of element edges or faces. Later on, the degrees of freedom of the CFE space will be exclusively assigned to the vertices of the (coarse) triangulation $\mathcal{T}$.

We consider the two triangulations $\mathcal{T}_{1}, \mathcal{T}_{2} \subset \mathcal{T}$,

$$
\mathcal{T}_{k}:=\left\{T \in \mathcal{T}: T \subset \bar{\Omega}_{k}\right\}, k=1,2,
$$

related to the subdomains. The union of these triangulations does not cover $\Omega$, in general. Some neighborhood of the interface, the interface zone

$$
\Omega^{\Gamma}:=\Omega \backslash\left(\left(\bigcup \mathcal{T}_{1}\right) \cup\left(\bigcup \mathcal{T}_{2}\right)\right),
$$

is not covered by elements of $\mathcal{T}_{1}$ or $\mathcal{T}_{2}$ unless the interface is resolved by $\mathcal{T}$. We introduce two triangulations of the interface zone, one associated with each subdomain. The elements $T \in \mathcal{T}$ that are contained in none of the two triangulations are collected in the set

$$
\mathcal{T}_{2}^{\Gamma}:=\mathcal{T} \backslash\left(\mathcal{T}_{1} \cup \mathcal{T}_{2}\right)
$$

A further fine triangulation $\mathcal{T}_{1}^{\Gamma}$ of $\Omega^{\Gamma}$ will be employed to adapt the shape of the ansatz functions in $\Omega_{1}$. This fine triangulation $\mathcal{T}_{1}^{\Gamma}$ is derived by regular refinement of $\mathcal{T}_{2}^{\Gamma}$ (e.g., by red-green-refinement or newest vertex bisection) locally near the interface. The corresponding $\mathcal{T}_{1}^{\Gamma}$-piecewise mesh width function $h_{1}^{\Gamma}: \bigcup \mathcal{T}_{1}^{\Gamma} \rightarrow \mathbb{R}_{>0}$ is given by

$$
h_{1}^{\Gamma}(x):=\max _{t \in \mathcal{T}_{1}^{\Gamma}: x \in t} \operatorname{diam}(t) .
$$

The refinement shall be done such that

$$
\left.h_{1}^{\Gamma}\right|_{t}=\operatorname{diam}(t) \geq C_{1}^{-1} \operatorname{dist}(t, \Gamma) \quad \text { for all } t \in \mathcal{T}_{1}^{\Gamma}
$$

holds with a universal constant $C_{1}$ independent of the $h_{1}^{\Gamma}$. This condition prevents over-refinement in the interface zone and enforces a certain grading of $\mathcal{T}_{1}^{\Gamma}$ toward the interface. This grading is essential for the stability, complexity, and accuracy of our method. The condition enters our error analysis via the external result [18, Theorem 4.4] which plays an essential role in the proof of Lemma 3.1 and, hence, in the proof of our main result Theorem 2.3

Note that condition (2.2) is satisfied with a constant $C_{1} \approx 2$ if the fine triangulation $\mathcal{T}_{1}^{\Gamma}$ is computed by successive refinement of those simplices that are intersected by $\Gamma$ (cf. [22, Section 2]). This shows that arbitrary small elements in a vicinity of the interface are possible in $\mathcal{T}_{1}^{\Gamma}$. Still, $\mathcal{T}_{1}^{\Gamma}$ is not aligned with $\Gamma$ in general. The analysis of Section 3 will show that the mesh size $\left.h^{\Gamma}\right|_{\Gamma}$ at the interface suffices to be of size $h^{3 / 2}$. This implies that the complexity of $\mathcal{T}_{1}^{\Gamma}$ depends only on the mesh size of the coarse mesh $\mathcal{T}$ and not on the location of the interface relative to the coarse mesh.

2.2.2. Additional structure. The meshes defined in the previous section cannot see the interface. However, precise information about the location of the interface is crucial for any reasonable approximation scheme. The exchange of information between the interface and the meshes shall be introduced via two mappings.

Closest inner simplex. The mapping $T_{(\cdot)}^{1}: V\left(\mathcal{T}_{1}^{\Gamma}\right) \rightarrow \mathcal{T}_{1}$ is chosen such that $T_{x}^{1} \in \operatorname{argmin}_{T \in \mathcal{T}_{1}} \operatorname{dist}(x, T)$, i.e., $T_{(\cdot)}^{1}$ assigns a closest inner simplex (fully contained in $\left.\Omega_{1}\right)$ to every vertex $x \in V\left(\mathcal{T}_{1}^{\Gamma}\right) . \quad \mathcal{I}_{T_{x}^{1}} u \in \mathbb{P}_{1}\left(\mathbb{R}^{2}\right)$ denotes the globally affine 


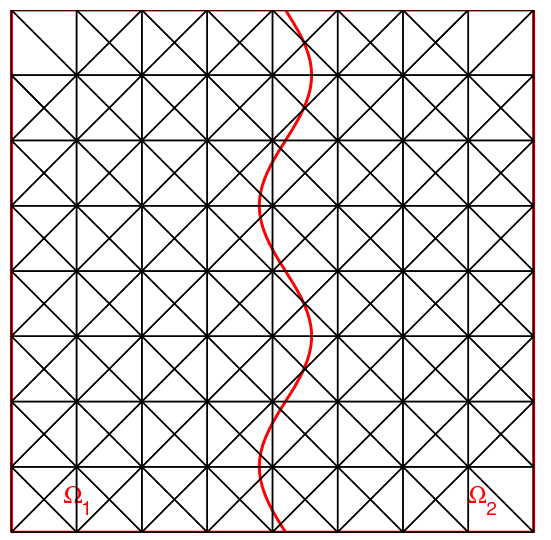

(a) The coarse triangulation $\mathcal{T}$.

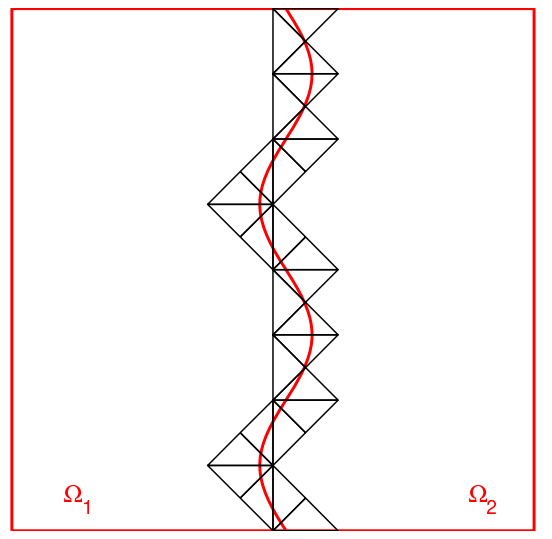

(b) The triangulation $\mathcal{T}_{2}^{\Gamma}$ of $\Omega^{\Gamma}$.

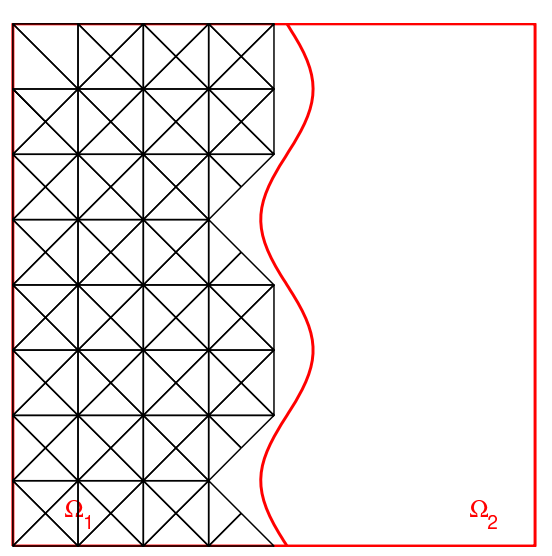

(d) The triangulation $\mathcal{T}_{1}$ related to $\Omega_{1}$.

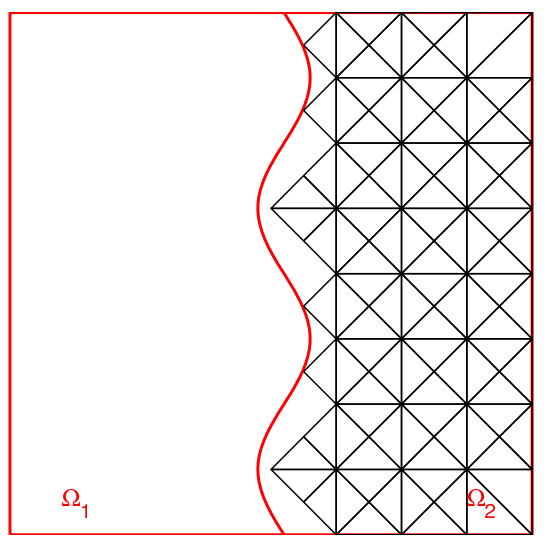

(c) The triangulation $\mathcal{T}_{2}$ related to $\Omega_{2}$. (e) The refined triangulation $\mathcal{T}_{1}^{\Gamma}$ of the interface zone $\Omega^{\Gamma}$.

Figure 1. The triangulations introduced in Section 2.2.1. The interface is not well represented in $\mathcal{T}$ shown in (a). It is better represented by $\mathcal{T}_{1}^{\Gamma}$ in (e) but still not resolved. 
function which interpolates $u$ in the vertices of $T_{x}^{1}$. Accordingly, $T_{(\cdot)}^{2}: V\left(\mathcal{T}_{2}^{\Gamma}\right) \rightarrow \mathcal{T}_{2}$ and $\mathcal{I}_{T_{x}^{2}} u$ are defined.

Interface projection. The projection operator $(\cdot)^{\Gamma}: \mathbb{R}^{d} \rightarrow \Gamma$ is chosen such that $x^{\Gamma} \in \operatorname{argmin}_{y \in \Gamma} \operatorname{dist}(x, y)$. This projection encodes the geometrical information about the interface that is required by our method.

2.2.3. The CFE space. By $S_{k}, k=1,2$, we denote the finite element space of continuous $\mathcal{T}_{k}$-piecewise affine functions

$$
S_{k}:=\left\{u: C^{0}\left(\bigcup \mathcal{T}_{k}\right):\left.u\right|_{T} \in \mathbb{P}^{1} \text { for all } T \in \mathcal{T}_{k},\left.u\right|_{\partial \Omega \cap \partial\left(\cup \mathcal{T}_{k}\right)}\right\}
$$

with the homogeneous Dirichlet boundary condition on $\partial \Omega$ built in. These spaces represent the degrees of freedom of the method, in that CFE shape functions are derived by extending elements from $S_{1}$ resp. $S_{2}$ to the interface zone.

In other words, CFE shape functions are certain elements of the target space

$$
\begin{aligned}
& S^{\Gamma}:=\left\{u \in H_{0}^{1}\left(\Omega_{1} \cap \Omega_{2}\right):\left.u\right|_{T \cap \Omega_{1}} \in \mathbb{P}^{1} \text { for all } T \in \mathcal{T}_{1} \cup \mathcal{T}_{1}^{\Gamma},\right. \\
& \left.\qquad\left.u\right|_{T \cap \Omega_{2}} \in \mathbb{P}^{1} \text { for all } T \in \mathcal{T}_{2} \cup \mathcal{T}_{2}^{\Gamma}\right\} .
\end{aligned}
$$

Definition of shape functions via extensions. The CFE space $S^{\text {cfe }}$ is given as the image of $S_{1} \times S_{2}$ under the bounded linear injective operator $\mathcal{P}^{\text {cfe }}: S_{1} \times S_{2} \rightarrow S^{\Gamma}$, i.e., $S^{\text {cfe }}:=\mathcal{P}^{\text {cfe }}(S)$. The definition of $\mathcal{P}^{\text {cfe }}$ is based on two mappings that relate the different meshes and the interface. The projection operator $\mathcal{P}^{\text {cfe }}$ is defined in the two subdomains as follows:

$$
\mathcal{P}^{\text {cfe }}\left(u_{1}, u_{2}\right)(x):= \begin{cases}\mathcal{P}_{1}^{\text {cfe }}\left(u_{1}, u_{2}\right)(x) & \text { if } x \in \Omega_{1} \\ \mathcal{P}_{2}^{\text {cfe }} u_{2}(x) & \text { if } x \in \Omega_{2}\end{cases}
$$

with $\mathcal{P}_{1}^{\text {cfe }}$ and $\mathcal{P}_{2}^{\text {cfe }}$ given subsequently.

Definition of $\mathcal{P}^{\mathrm{cfe}_{2}}$. The operator $\mathcal{P}_{2}^{\text {cfe }}$ extends functions defined in $\bigcup \mathcal{T}_{2}$ to the interface zone $\Omega \Gamma$. Given $u_{2} \in S_{2}$, the continuous $\left(\mathcal{T}_{2} \cup \mathcal{T}_{2}^{\Gamma}\right)$-piecewise affine function $\mathcal{P}_{2}^{\text {cfe }} u_{2}$ is uniquely defined by nodal values

$$
\left(\mathcal{P}_{2}^{\text {cfe }} u_{2}\right)(x):= \begin{cases}u_{2}(x) & \text { if } x \in V\left(\mathcal{T}_{2}\right), \\ \mathcal{I}_{T_{x}^{2}} u(x) & \text { if } x \in V\left(\mathcal{T}_{2}^{\Gamma}\right) \backslash V\left(\mathcal{T}_{2}\right) \text { and } x \notin \partial \Omega^{\Gamma} \cap \partial \Omega, \\ 0 & x \in \partial \Omega^{\Gamma} \cap \partial \Omega\end{cases}
$$

with $\mathcal{I}_{T_{x}^{2}} u$ defined in Section 2.2 .2 above.

Definition of $\mathcal{P}^{\text {cfe }}{ }_{1}$. The operator $\mathcal{P}_{1}^{\text {cfe }}$ extends functions defined in $\bigcup \mathcal{T}_{1}$ to the interface zone $\Omega^{\Gamma}$ in such a way that its trace on $\Gamma$ approximately coincides with $\left.\left(\mathcal{P}_{2}^{\text {cfe }} u_{2}\right)\right|_{\Gamma}$. Given $u_{1} \in S_{1}, u_{2} \in S_{2}, \mathcal{P}_{1}^{\text {cfe }}\left(u_{1}, u_{2}\right)$ is the unique continuous $\left(\mathcal{T}_{1} \cup \mathcal{T}_{1}^{\Gamma}\right)$ piecewise affine function which takes the following values at vertices $x \in V\left(\mathcal{T}_{1} \cup \mathcal{T}_{1}^{\Gamma}\right)$ :

$$
\begin{aligned}
& \mathcal{P}_{1}^{\text {cfe }}\left(u_{1}, u_{2}\right)(x) \\
& :=\left\{\begin{array}{lc}
u_{1}(x) & \text { if } x \in V\left(\mathcal{T}_{1}\right) \\
& \text { or } x \in V\left(\mathcal{T}_{1}^{\Gamma}\right) \cap\left(\bigcup \mathcal{T}_{1}\right), \\
\left.\mathcal{P}_{2}^{\text {cfe }} u_{2}\right)\left(x^{\Gamma}\right)+\left\langle\nabla \mathcal{I}_{T_{x}^{1}} u_{1}, x-x^{\Gamma}\right\rangle & \text { if } x \in V\left(\mathcal{T}_{1}^{\Gamma}\right) \backslash\left(\bigcup \mathcal{T}_{1}\right) \\
& \text { and } x \notin \partial \Omega^{\Gamma} \cap \partial \Omega, \\
0 & \text { if } x \in \partial \Omega^{\Gamma} \cap \partial \Omega
\end{array}\right.
\end{aligned}
$$


with $\mathcal{I}_{T_{x}^{1}} u$ defined in Section 2.2 .2 above. Note that the definition of $\mathcal{P}_{1}^{\text {cfe }}$ ensures continuity of its images although $\mathcal{T}_{1} \cap \mathcal{T}_{1}^{\Gamma}$ is not necessarily a regular triangulation in the sense that hanging nodes may appear (as in Figure 1(d)-(e)).

Although the one-dimensional case ( $\Omega$ is an interval and $\Gamma$ is some point in $\Omega$ ) does not share the numerical difficulties of the multi-dimensional setting (because the interface can easily be resolved by adding the vertex $\Gamma$ to any mesh), it clearly illustrates the definition of $\mathcal{P}^{\text {cfe }}$ and the derivation of our shape functions (see Figures 2 23). Note that, in one dimension, our construction ensures continuity across the interface and the method is conforming. In general, conformity is only achieved in the limit $\left.h_{1}^{\Gamma}\right|_{\Gamma} \rightarrow 0$. However, the discontinuity of shape functions across the interface (see Figure (4) is sufficiently small to preserve stability and accuracy of our method.

Remark 2.1. There is some algorithmic freedom in the above construction:

(1) It is not essential that the subtriangulations $\mathcal{T}_{1}, \mathcal{T}_{2}, \mathcal{T}_{2}^{\Gamma}$ form a partition of some regular triangulation $\mathcal{T}$. They could have been chosen to be nonmatching overlapping triangulations representing $\Omega_{1}, \Omega_{2}$, and some neighborhood $\Omega^{\Gamma}$ of the interface $\Gamma$.

(2) It is not essential that the definitions of the mappings $T_{(\cdot)}^{1}$ and $(\cdot)^{\Gamma}$ in the above construction are based on the minimality of certain distances; any point or simplex sufficiently close (distance proportional to local mesh size) would do the job as well.

2.2.4. A local basis of the CFE space. The degrees of freedom of the method are function values at vertices

$$
V_{\text {dof }}(\mathcal{T}):=V\left(\mathcal{T}_{1}\right) \cup V\left(\mathcal{T}_{2}\right) \subset V(\mathcal{T}) .
$$

Hence, degrees of freedom are solely assigned to vertices in the coarse (interface independent) mesh $\mathcal{T}$ and every vertex in $\mathcal{T}$ represents at most one basis function of $S^{\mathrm{cfe}}$.

The images of the nodal basis functions $\lambda_{z} \in S_{1} \cup S_{2}$ for $z \in V_{\text {dof }}(\mathcal{T})$ yield a basis of $S^{\mathrm{cfe}}$, i.e.,

$$
S^{\text {cfe }}=\operatorname{span}\left(\left\{\mathcal{P}^{\text {cfe }}\left(\lambda_{z}, 0\right) \mid z \in V\left(\mathcal{T}_{1}\right)\right\} \cup\left\{\mathcal{P}^{\text {cfe }}\left(0, \lambda_{z}\right) \mid z \in V\left(\mathcal{T}_{2}\right)\right\}\right),
$$

where $\mathcal{P}^{\text {cfe }} \lambda_{z}$ and $\mathcal{P}^{\text {cfe }} \lambda_{y}$ are linearly independent if $z \neq y$.

Most of the basis functions are standard nodal basis functions. More precisely, $\mathcal{P}^{\text {cfe }}$ has no effect on functions that vanish in $\Omega^{\Gamma}$ plus one layer of coarse elements $T \in \mathcal{T}$. Only a few basis functions are manipulated via the explicit linear operator $\mathcal{P}^{\text {cfe }}$. Those basis functions have slightly enlarged supports when compared with standard nodal basis functions on $\mathcal{T}$. However, the supports remain local in the sense that their diameters remain proportional to the local coarse mesh size $h$. Thus, the supports have finite overlap independent of the mesh size $h$.

2.3. Discrete problem. The discrete variational formulation of (2.1) reads: Find $u^{\text {cfe }} \in S^{\text {cfe }}$ such that

$$
\int_{\Omega} a \nabla u^{\text {cfe }} \cdot \nabla v \mathrm{~d} x=\int_{\Omega} f v \mathrm{~d} x \quad \text { for all } v \in S^{\text {cfe }} .
$$

Note that the basis given in the previous section turns this variational problem into a system of linear algebraic equations. Since those basis functions have local support, sparsity of the corresponding stiffness matrix is ensured. 


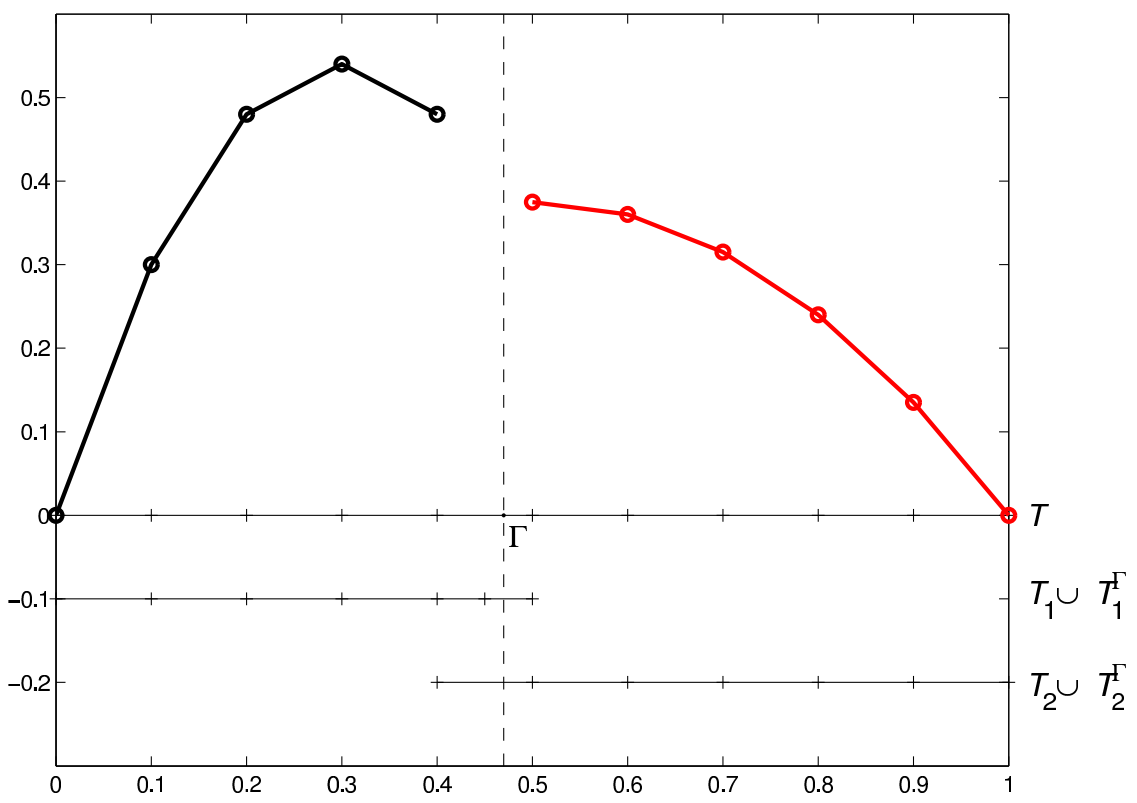

(a) Some functions $u_{1} \in S_{1}$ (left) and $u_{2} \in S_{2}$ (right) representing the degrees of freedom.

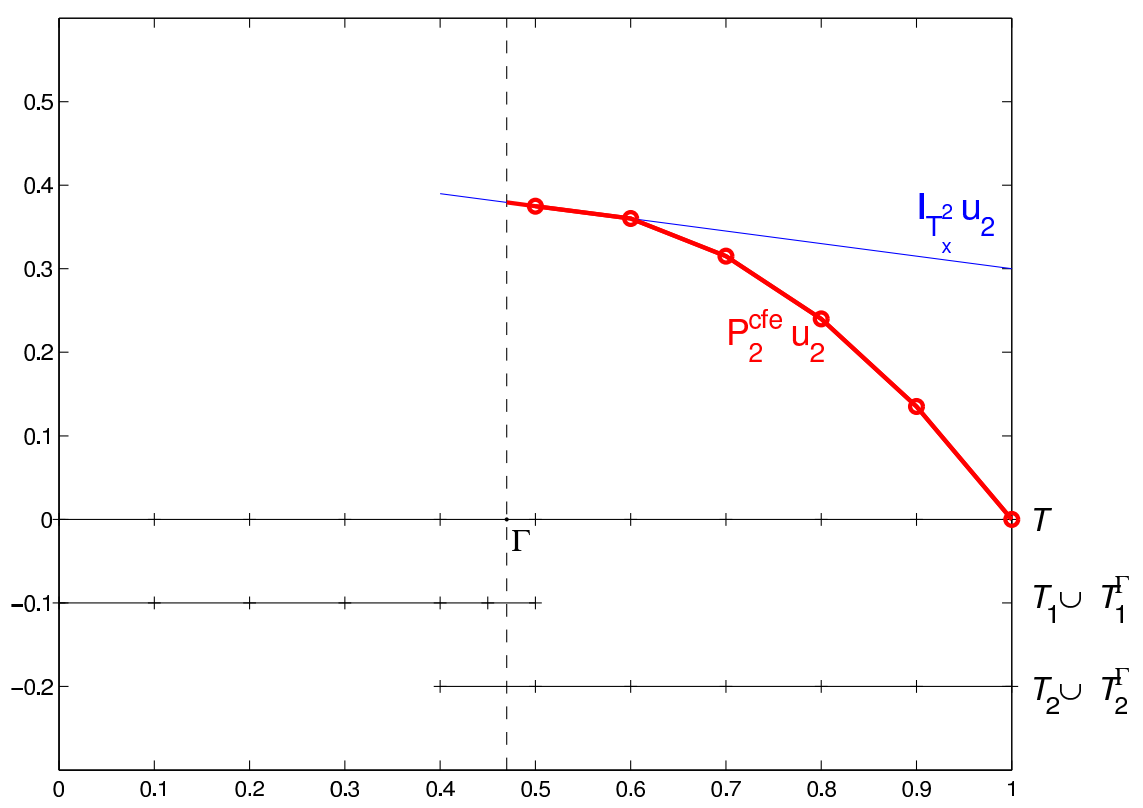

(b) Extension $\mathcal{P}_{2}^{\text {cfe }} u_{2}$ of $u_{2}$ to the interface zone $\Omega^{\Gamma}$.

Figure 2. Illustration of the definition of the CFE space in Section 2.2 .3 


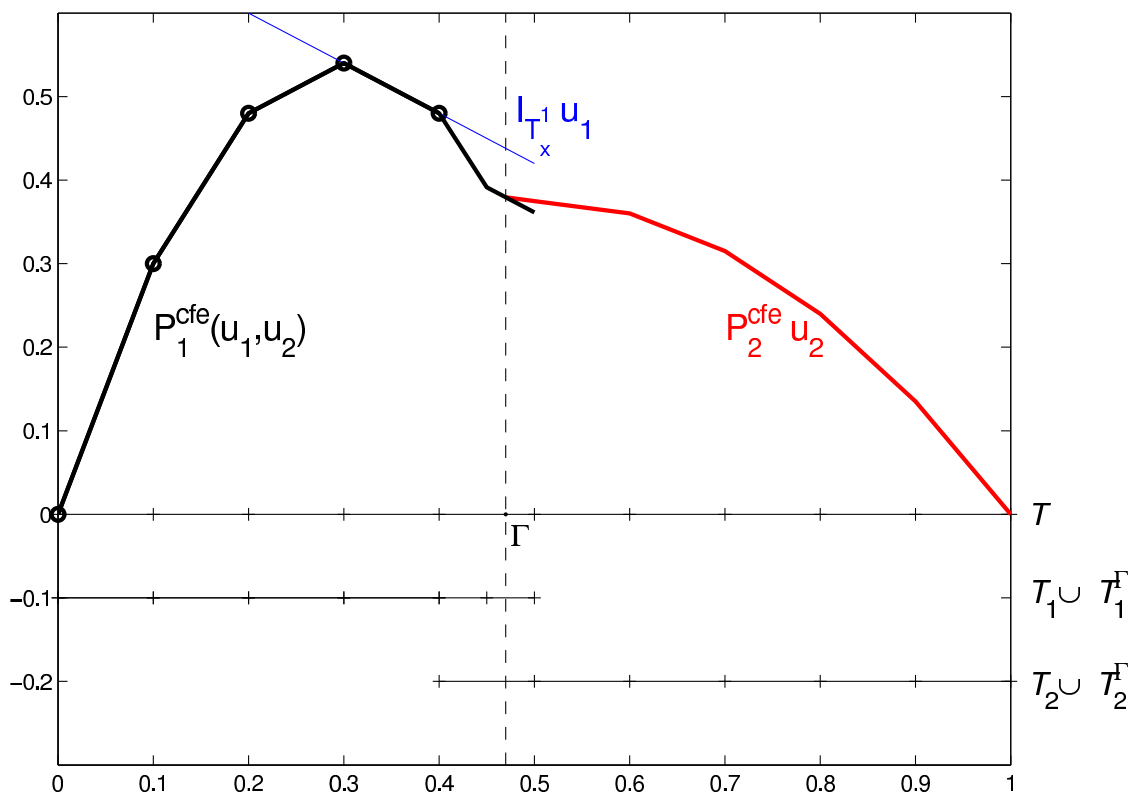

(a) Extension $\mathcal{P}_{1}^{\text {cfe }} u_{1}$ of $u_{1}$ to the interface zone $\Omega^{\Gamma}$.

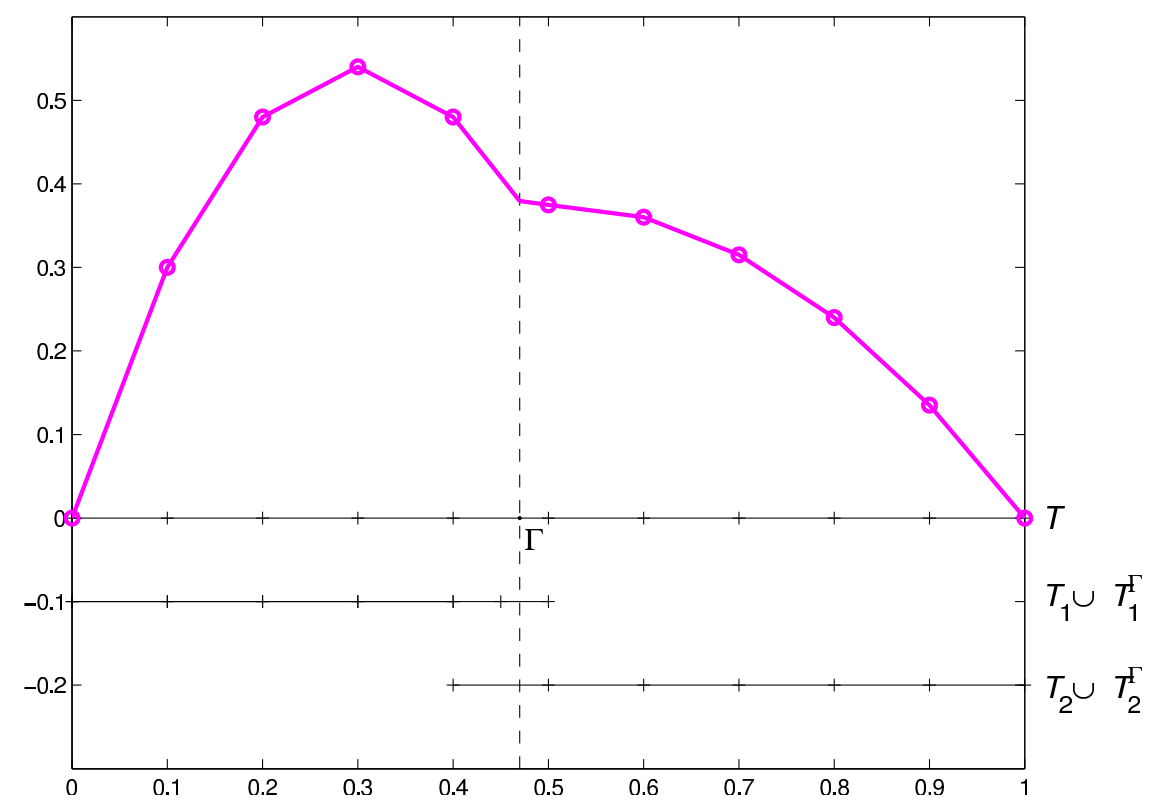

(b) The corresponding CFE function $\mathcal{P}^{\text {cfe }}\left(u_{1}, u_{2}\right) \in S^{\text {cfe }}$ with degrees of freedom (o).

Figure 3. Illustration (continued from Figure 2) of the definition of the CFE space in Section 2.2.3. 


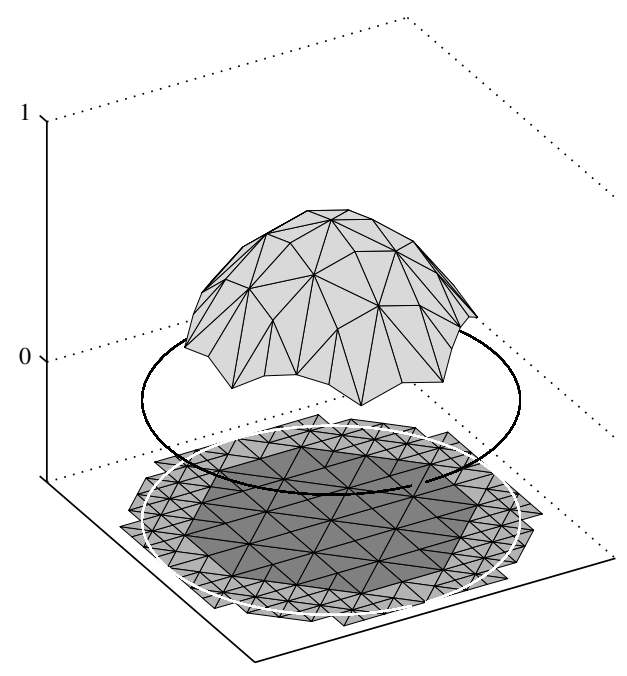

(a) Some function $u_{1} \in S_{1}$ defined on $\bigcup \mathcal{T}_{1}$.

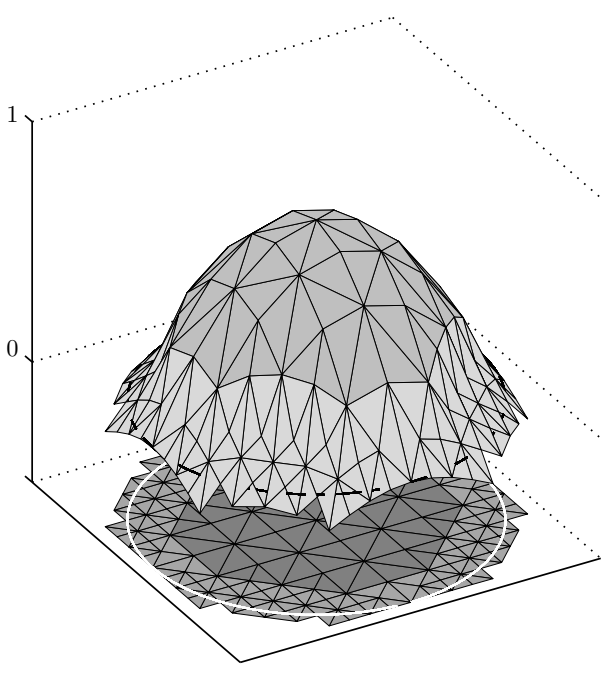

(b) Extension $\mathcal{P}_{1}^{\text {cfe }}\left(u_{1}, 0\right)$ of $u_{1}$.

FIGURE 4. Illustration of the approximate trace matching across the interface: $\Gamma$ is the unit circle and $\Omega_{1}$ its interior, $u_{2}$ and, hence, $\left.\mathcal{P}_{2}^{\text {cfe }} u_{2}\right|_{\Gamma}$ are chosen zero.

Remark 2.2. The implementation of the method is similar to previous CFE methods and we refer to [7,16,21,22, for computational insights.

One issue is that the solution of (2.8) requires the evaluation of integrals over intersections $T \cap \Omega_{k}$ which is beyond the scope of this note. The forthcoming theoretical results assume that all integrals are evaluated exactly. We refer to [7, 15, 22 for a practical resolution of this issue.

2.4. Error estimates. The following theorem addresses the solvability of (2.8). Moreover, assuming $H^{2}\left(\Omega_{1} \cup \Omega_{2}\right)$-regularity, an optimal a priori error bound in energy norm is given. Besides parameters already mentioned in the construction, the constant in the error estimate depends on $\rho_{T}$, which is the ratio between the diameter of the largest ball that can be inscribed in $T \in \mathcal{T}$ and $\operatorname{diam}(T)$. The triangulations $\mathcal{T}$ and $\mathcal{T}_{1}^{\Gamma}$ are assumed to be non-degenerate, i.e., $\rho_{\mathcal{T}}:=\min _{T \in \mathcal{T}} \rho_{T}>0$ (resp. $\rho_{\mathcal{T}_{1}^{\Gamma}}:=\min _{T \in \mathcal{T}_{1}^{\Gamma}} \rho_{T}>0$ ).

Theorem 2.3 (Linear convergence with respect to mesh size). The discrete problem (2.8) always has a unique solution $u^{\text {cfe }} \in S^{\text {cfe }}$.

If, in addition, the solution of (2.1), $u^{\star} \in H_{0}^{1}(\Omega)$, is piecewise smooth, $u^{\star} \in$ $H^{2}\left(\Omega_{1} \cup \Omega_{2}\right)$, and if

$$
\left\|h_{1}^{\Gamma} / h^{3 / 2}\right\|_{L^{\infty}\left(\bigcup\left\{t \in \mathcal{T}_{1}^{\Gamma}: t \cap \Gamma \neq \emptyset\right\}\right)} \leq C_{2}
$$

for some generic constant $C_{2}$, then the following a priori error estimate holds:

$$
\left\|\left|u^{\star}-u^{\mathrm{cfe}}\right|\right\| \leq C\|h\|_{L^{\infty}(\Omega)}\left\|\sqrt{a} \nabla^{2} u\right\|_{L^{2}\left(\Omega_{1} \cup \Omega_{2}\right)} .
$$

The constant $C=C\left(\rho_{\mathcal{T}}, \rho_{\mathcal{T}_{1}^{\Gamma}}, C_{1}, C_{2}\right)$ does not depend on the mesh width functions $h, h^{\Gamma}$ and the contrast parameter $a_{\text {cont }}$. 
Proof. The unique solvability of (2.8) follows from the fact that $\||\cdot|||$ is a norm in $S^{\text {cfe }}$. Since, in the limit $\left.h_{1}^{\Gamma}\right|_{\Gamma} \rightarrow 0, S^{\text {cfe }}$ is conforming, the latter is quite obvious if $\left.h_{1}^{\Gamma}\right|_{\left(\bigcup\left\{t \in \mathcal{T}_{1}^{\Gamma}: t \cap \Gamma \neq \emptyset\right\}\right)}$ is sufficiently small. Otherwise, this property can be proven along the lines of [18, Lemma 4.10].

The proof of the error estimate will be given in Section 3

The error estimate in the above theorem rests on the regularity of the solution $u^{\star} \in H^{2}\left(\Omega_{1} \cup \Omega_{2}\right)$. In general, this regularity does not hold for solutions of problem (2.1). Moreover, even though the constant in the error estimate does not depend on the contrast $a_{\text {cont }}$, the $H^{2}\left(\Omega_{1} \cup \Omega_{2}\right)$ seminorm of the solution on the right-hand side of estimate (2.10) may do. In Section 4 we will prove that $f \in L^{2}(\Omega)$, the Lipschitz properties of the subdomains $\Omega_{k}$, and, in addition, convexity of $\Omega \subset \mathbb{R}^{2}$ and the assumption $\Gamma \in C^{1,1}$ imply $u^{\star} \in H^{2}\left(\Omega_{1} \cup \Omega_{2}\right)$ and

$$
\left\|\nabla^{2} u^{\star}\right\|_{L^{2}\left(\Omega_{1}\right)} \leq C_{\mathrm{reg}}\|f\|_{L^{2}(\Omega)}, \quad\left\|\nabla^{2} u^{\star}\right\|_{L^{2}\left(\Omega_{2}\right)} \leq \frac{C_{\mathrm{reg}}}{a_{\text {cont }}}\|f\|_{L^{2}(\Omega)}
$$

with some universal constant $C_{\text {reg }}$ that depends only on the geometry of the subdomains $\Omega_{k}$ and the interface $\Gamma$ but not on $f$ and $a_{\text {cont }}$. Hence, under those assumptions on the geometry, the error of the CFE method does not depend on the contrast parameter $a_{\text {cont }}$.

Theorem 2.4 (Contrast independence). If $\Omega \subset \mathbb{R}^{2}$ is convex, $\Gamma \in C^{1,1}$, and if (2.9) is satisfied, then the following a priori error estimate holds:

$$
\left\|u^{\star}-u^{\mathrm{cfe}} \mid\right\| \leq C\|h\|_{L^{\infty}(\Omega)}\|f\|_{L^{2}(\Omega)} .
$$

The constant $C=C\left(\rho_{\mathcal{T}}, \rho_{\mathcal{T}_{1}^{\Gamma}}, C_{1}, C_{2}, C_{\mathrm{reg}}\right)$ does not depend on $f$, the mesh width functions $h, h_{1}^{\Gamma}$, and the contrast $a_{\mathrm{cont}}$.

Remark 2.5. As already mentioned in the introduction, our method is designed to capture the kink of the solution across the interface. Further lack of regularity, caused, e.g., by singularities at kinks of the interface, is not addressed by the proposed method and leads to reduced convergence rates. The actual rate depends on the strength of the singularities as usual, i.e., if $u^{\star} \in H^{1+s}\left(\Omega_{1} \cup \Omega_{2}\right)$ for some $s \in$ $[0,1[$, then standard interpolation theory of Sobolev spaces allows one to estimate

$$
\left\|u^{\star}-u^{\mathrm{cfe}}\right\| \leq C\|h\|_{L^{\infty}(\Omega)}^{s} .
$$

Standard techniques may be applied to improve the convergence rate of the method for singular solutions, e.g., adding certain singular functions to the approximation space, or adaptive refinement of the coarse mesh $\mathcal{T}$ toward the singularity.

2.5. Complexity. Let us briefly discuss the complexity of our method. Considering a uniform coarse-scale grid of width $h$, the number of degrees of freedom in our method is proportional to $h^{-d}$, where $d \in\{2,3\}$ denotes the dimension of the physical space as before. The cost of setting up and storing the basis functions can be estimated as follows. Because of Theorems 2.3 and 2.4, it is sufficient to adapt the shape functions on a submesh with minimal mesh size $\left.h_{1}^{\Gamma}\right|_{\Gamma} \approx h^{3 / 2}$ close to the interface. Since the dimension of the interface $\Gamma$ is $d-1$ and owing to (2.2), the number of elements in the submesh is proportional to $h^{-3 / 2(d-1)}$, i.e., $h^{-3 / 2}$ for $d=2$ and $h^{-3}$ for $d=3$. Hence, the cost caused by the adaptation of the shape functions is at most proportional to the number of degrees of freedom $h^{-d}$. 


\section{Detailed ERror ANALysis}

This section proves the error estimate in Theorem 2.3. The error of the CFE approximation can be estimated as in [3, Lemma 10.1.7] by

$$
\left|\left\|u^{\star}-u^{\mathrm{cfe}}||\left|\leq \inf _{v \in S^{\mathrm{cfe}}}\right||| u^{\star}-v \mid\right\|+\sup _{0 \neq v \in S^{\mathrm{cfe}}} \frac{\left|\mathfrak{a}\left(u^{\star}-u^{\mathrm{cfe}}, v\right)\right|}{|| v|\||} .\right.
$$

The first term in the above estimate reflects the best approximation error which is further addressed in Section 3.1. The additional second term is due to nonconformity (see Section 3.2).

3.1. Approximation property. For $\mathcal{G} \in\left\{\mathcal{T}, \mathcal{T}_{1}, \mathcal{T}_{2}, \mathcal{T}_{1}^{\Gamma}, \mathcal{T}_{2}\right\}$, let $\mathcal{I}_{\mathcal{G}} u$ denote the unique $\mathcal{G}$-piecewise affine function that interpolates a sufficiently smooth function $u$ at the vertices of $\mathcal{G}$. The solution $u^{\star} \in H^{2}\left(\Omega_{1} \cup \Omega_{2}\right)$ of (2.1) is well approximated by the discontinuous function $u_{h}$ with $\left.u_{h}\right|_{\Omega_{k}}=\left.\left(\mathcal{I}_{\mathcal{T}_{k}} u\right)\right|_{\Omega_{k}}, k=1,2$. The error in the energy norm is proportional to $h$. This approximation property is preserved if $u_{h}$ is suitably mapped onto the finite element space $S^{\text {cfe }}$ as the following lemma states.

For the ease of notation, observe that $\mathcal{P}^{\text {cfe }} u:=\mathcal{P}^{\text {cfe }}\left(\mathcal{I}_{\mathcal{T}_{1}} u, \mathcal{I}_{\mathcal{T}_{2}} u\right)$ defines $\mathcal{P}^{\text {cfe }}$ for arguments $u \in H_{0}^{1}(\Omega) \cap H^{2}\left(\Omega_{1} \cup \Omega_{2}\right)$. Accordingly, $\mathcal{P}_{2}^{\text {cfe }} u:=\mathcal{P}_{2}^{\text {cfe }} \mathcal{I}_{\mathcal{T}_{2}} u$ (resp. $\left.\mathcal{P}_{1}^{\text {cfe }} u:=\mathcal{P}_{1}^{\text {cfe }}\left(\mathcal{I}_{\mathcal{T}_{1}} u, \mathcal{I}_{\mathcal{T}_{2}} u\right)\right)$ extends $\mathcal{P}_{2}^{\text {cfe }}\left(\right.$ resp. $\left.\mathcal{P}_{1}^{\text {cfe }}\right)$ to $H_{0}^{1}(\Omega) \cap H^{2}\left(\Omega_{1} \cup \Omega_{2}\right)$.

Lemma 3.1 (Approximation property of $S^{\mathrm{cfe}}$ ). There is a constant $C>0$ which may depend on $\rho_{\mathcal{T}}, \rho_{\mathcal{T}_{1}^{\Gamma}}, C_{1}, C_{2}$ but not on $h$ and $h_{1}^{\Gamma}$ such that for all $u \in H_{0}^{1}(\Omega) \cap$ $H^{2}\left(\Omega_{1} \cup \Omega_{2}\right)$ it holds that

$$
\left\|u-\mathcal{P}^{\mathrm{cfe}} u\right\|\|\leq C\| \sqrt{a} h \nabla^{2} u \|_{L^{2}\left(\Omega_{1} \cup \Omega_{2}\right)} .
$$

Proof. The proof picks up some standard techniques for CFEs as they are used, e.g., in the proof of Theorem 4.4 in 18 . In addition, we will frequently make use of classical error estimates of nodal interpolation with respect to simplices. Following [5. Theorem 16.1], there exists a universal constant $C_{\text {ip }}$ such that

$$
\left|u-\mathcal{I}_{t} u\right|_{W_{p}^{m}(t)} \leq \frac{C_{\mathrm{ip}}}{\rho_{t}} \operatorname{diam}(t)^{2-\frac{d}{2}-\frac{d}{p}-m}|u|_{H^{2}(t)}
$$

for all $u \in H^{2}(t), m \in\{0,1\}$, provided $W_{p}^{m}(t) \subset H^{2}(t) ; \mathcal{I}_{t} u$ denotes the affine interpolant of $u$ at the vertices of a triangle $t$.

The main tool for exploiting the piecewise regularity is a suitable extension operator. It is known that, since $\Omega_{k}$ is assumed bounded and Lipschitz, there exists a continuous, linear extension operator $\mathfrak{E}_{k}: H^{2}\left(\Omega_{k}\right) \cap H_{0}^{1}(\Omega) \rightarrow H^{2}(\Omega) \cap H_{0}^{1}(\Omega)$, $k \in\{1,2\}$, such that for all $u \in H^{2}\left(\Omega_{k}\right) \cap H_{0}^{1}(\Omega)$ there holds

$$
\left.\mathfrak{E}_{k} u\right|_{\Omega_{k}}=u \text { and }\left\|\nabla^{2} \mathfrak{E} u\right\|_{L^{2}(\Omega)} \leq C_{\text {ext }}\left\|\nabla^{2} u\right\|_{L^{2}\left(\Omega_{k}\right)}
$$

with a constant $C_{\text {ext }}$ that depends only on $\Omega_{k}$ and $\Omega$ [25]. Moreover, $C_{\text {ext }}$ is moderately small under mild assumptions on the geometry 23. Throughout the rest of the paper, $u_{k}$ abbreviates $\mathfrak{E}_{k} u, k=1,2$.

Our proof rests upon the splitting

$$
u=u_{2}+\left(u-u_{2}\right)
$$


and the observation that $\left.\left(u-u_{2}\right)\right|_{\Omega_{1}} \in H_{0}^{1}\left(\Omega_{1}\right) \cap H^{2}\left(\Omega_{1}\right)$ and $\left.\left(u-u_{2}\right)\right|_{\Omega_{2}}=0$. The splitting and the linearity of $\mathcal{P}^{\text {cfe }}$ lead to the upper bound

$$
\left\|\left|u-\mathcal{P}^{\mathrm{cfe}} u\left\|\left.\right|^{2} \leq\right\|\left\|u_{2}-\mathcal{P}^{\mathrm{cfe}} u_{2}\right\|\right|^{2}+\right\| \nabla\left(u-u_{2}-\mathcal{P}^{\mathrm{cfe}}\left(u-u_{2}\right)\right) \|_{L^{2}\left(\Omega_{1}\right)}^{2} .
$$

The second term on the right-hand side of (3.4) can be bounded by classical techniques for the analysis of CFEs. In particular, [18, Theorem 4.4] and (3.3) show that

$$
\left\|\nabla\left(\left(u-u_{2}\right)-\mathcal{P}^{\mathrm{cfe}}\left(u-u_{2}\right)\right)\right\|_{L^{2}\left(\Omega_{1}\right)}^{2} \leq C\left\|h \nabla^{2} u\right\|_{H^{2}\left(\Omega_{1}\right)}
$$

with some constant $C$ that depends only on $\rho_{\mathcal{T}}, \rho_{\mathcal{T}_{1}^{\Gamma}}, C_{1}, C_{2}$, and $C_{\text {ext }}$.

Thus, we are left to bound the first term on the right-hand side of (3.4). The advantage of the splitting is that, compared to the initial assertion, we can now make use of the fact that $u_{2} \in H^{2}(\Omega)$ regardless of the interface.

Throughout the rest of the proof, $a \lesssim b$ abbreviates $a \leq C b$ with some constant $C$ that depends only on the constants $C_{1}, C_{2}, C_{\text {ip }}, C_{\text {ext }}, \rho_{\mathcal{T}}$, and $\rho_{\mathcal{T}_{1}^{\Gamma}}$.

By repeated use of the triangle inequality we separate the elements where standard estimates apply from those where more involved techniques are required:

$$
\begin{aligned}
& \left.\left\|u_{2}-\mathcal{P}^{\mathrm{cfe}} u_{2}\right\|\right|^{2}=\left\|\nabla\left(u_{2}-\mathcal{P}^{\mathrm{cfe}} u_{2}\right)\right\|_{L^{2}\left(\Omega_{1}\right)}^{2}+a_{\text {cont }}\left\|\nabla\left(u_{2}-\mathcal{P}^{\mathrm{cfe}} u_{2}\right)\right\|_{L^{2}\left(\Omega_{2}\right)}^{2} \\
& \stackrel{[2.6], 2.7]}{\leq}\left\|\nabla\left(u_{2}-\mathcal{I}_{\mathcal{T}} u_{2}\right)\right\|_{L^{2}\left(\Omega_{1}\right)}^{2}+a_{\text {cont }}\left\|\nabla\left(u_{2}-\mathcal{I}_{\mathcal{T}} u_{2}\right)\right\|_{L^{2}\left(\Omega_{2}\right)}^{2} \\
& +\left\|\nabla\left(\mathcal{I}_{\mathcal{T}} u_{2}-\mathcal{P}_{1}^{\text {cfe }} u_{2}\right)\right\|_{L^{2}\left(\Omega^{\Gamma}\right)}^{2}+a_{\text {cont }}\left\|\nabla\left(\mathcal{I}_{\mathcal{T}} u_{2}-\mathcal{P}_{2}^{\text {cfe }} u_{2}\right)\right\|_{L^{2}\left(\Omega^{\Gamma}\right)}^{2} \\
& \stackrel{\text { (3.2), (3.3) }}{\lesssim}\left\|h \nabla^{2} u_{2}\right\|_{L^{2}\left(\Omega_{1}\right)}^{2}+a_{\text {cont }}\left\|h \nabla^{2} u_{2}\right\|_{L^{2}\left(\Omega_{2}\right)}^{2} \\
& +\left\|\nabla\left(\mathcal{I}_{\mathcal{T}} u_{2}-\mathcal{P}_{1}^{\mathrm{cfe}} u_{2}\right)\right\|_{L^{2}\left(\Omega^{\Gamma}\right)}^{2}+a_{\text {cont }}\left\|\nabla\left(\mathcal{I}_{\mathcal{T}} u_{2}-\mathcal{P}_{2}^{\text {cfe }} u_{2}\right)\right\|_{L^{2}\left(\Omega^{\Gamma}\right)}^{2} .
\end{aligned}
$$

Let $t \in \mathcal{T}_{1}^{\Gamma}$ and $T \in \mathcal{T}_{2}^{\Gamma}$ such that $t \subset T$ (recall that $\mathcal{T}_{1}^{\Gamma}$ was derived from $\mathcal{T}_{2}^{\Gamma} \subset \mathcal{T}$ by refinement). Then, by an inverse estimate,

$$
\left\|\nabla\left(\mathcal{I}_{T} u_{2}-\mathcal{P}_{1}^{\text {cfe }} u_{2}\right)\right\|_{L^{2}(t)} \leq 2 \operatorname{diam}(t)^{d / 2} \operatorname{diam}(T)^{-1}\left\|\mathcal{I}_{T} u_{2}-\mathcal{P}_{1}^{\text {cfe }} u_{2}\right\|_{L^{\infty}(t)} .
$$

We fix $x \in V(t)$ with $\left\|\mathcal{I}_{T} u_{2}-\mathcal{P}_{1}^{\text {cfe }} u_{2}\right\|_{L^{\infty}(t)}=\left|\mathcal{I}_{T} u_{2}(x)-\mathcal{P}_{1}^{\text {cfe }} u_{2}(x)\right|$ and define $T_{t}^{1}:=T_{x}^{1}, T_{t}^{2}:=T_{x}^{2}$. In addition we introduce neighborhoods

$$
\omega_{T}:=\bigcup\{K \in \mathcal{T}: T \cap K \neq \emptyset\}
$$

containing both coarse elements $T_{t}^{1}$ and $T_{t}^{2}$. The definition of $\mathcal{P}_{1}^{\text {cfe }}$ in (2.7) and the application of Lemma 4.1 from 18 lead to

$$
\begin{aligned}
& \left\|\mathcal{I}_{T} u_{2}-\mathcal{P}_{1}^{\text {cfe }} u_{2}\right\|_{L^{\infty}(t)} \stackrel{\sqrt{2.7}}{=}\left|\mathcal{I}_{T} u_{2}(x)-\mathcal{I}_{T_{t}^{2}} u_{2}\left(x^{\Gamma}\right)-\left\langle\nabla \mathcal{I}_{T_{t}^{1}} u_{2}, x-x^{\Gamma}\right\rangle\right| \\
& \left.\lesssim\left|\mathcal{I}_{T} u_{2}(x)-\mathcal{I}_{T_{t}^{2}} u_{2}(x)\right|+\mid\left\langle\nabla\left(\mathcal{I}_{T_{t}^{2}} u_{2}-\mathcal{I}_{T_{t}^{1}} u_{2}\right), x-x^{\Gamma}\right\rangle\right) \mid \\
& \lesssim \operatorname{diam}(T)^{-d / 2}\left(\left\|\mathcal{I}_{T} u_{2}-\mathcal{I}_{T_{t}^{2}} u_{2}\right\|_{L^{2}(T)}+\operatorname{diam}(t)\left\|\nabla\left(\mathcal{I}_{T} u_{2}-\mathcal{I}_{T_{t}^{2}} u_{2}\right)\right\|_{L^{2}(T)}\right. \\
& \left.+\operatorname{diam}(t)\left\|\nabla\left(\mathcal{I}_{T_{t}^{2}} u_{2}-\mathcal{I}_{T_{t}^{1}} u_{2}\right)\right\|_{L^{2}(T)}\right) \\
& \stackrel{\operatorname{diam}(t) \leq \operatorname{diam}(T)}{\lesssim} \operatorname{diam}(T)^{2-d / 2}\left\|\nabla^{2} u_{2}\right\|_{L^{2}\left(\omega_{T}\right)} \text {. }
\end{aligned}
$$


The summation over all $t \in \mathcal{T}_{1}^{\Gamma}$ yields

$$
\begin{aligned}
& \left\|\nabla\left(\mathcal{I}_{\mathcal{T}} u_{2}-\mathcal{P}_{1}^{\mathrm{cfe}} u_{2}\right)\right\|_{L^{2}\left(\Omega^{\Gamma}\right)}^{2} \leq \sum_{t \in \mathcal{T}_{1}^{\Gamma}}\left\|\nabla\left(\mathcal{I}_{t} u_{2}-\mathcal{P}_{1}^{\mathrm{cfe}} u_{2}\right)\right\|_{L^{2}(t)}^{2} \\
& \leq \sum_{T \in \mathcal{T}} \sum_{t \in \mathcal{T}_{1}^{\Gamma}: t \subset T}\left\|\nabla\left(\mathcal{I}_{T} u_{2}-\mathcal{P}_{1}^{\mathrm{cfe}} u_{2}\right)\right\|_{L^{2}(t)}^{2} \\
& \stackrel{\sqrt[3.7]{3.8)}}{\lesssim} \sum_{T \in \mathcal{T}}\left(\sum_{t \in \mathcal{T}_{1}^{\Gamma}: t \subset T}|t|\right) \operatorname{diam}(T)^{2-d}\left\|\nabla^{2} u_{2}\right\|_{L^{2}\left(\omega_{T}\right)}^{2} \\
& \stackrel{\sqrt[3.3]{\vdots}}{\lesssim}\left\|h \nabla^{2} u\right\|_{L^{2}\left(\Omega_{2}\right)}^{2}
\end{aligned}
$$

Similar arguments as in (3.7), (3.8), and (3.9) lead to an estimate of the last term on the right-hand side of (3.6),

$$
\left\|\nabla\left(\mathcal{I}_{\mathcal{T}_{2}} u_{2}-\mathcal{P}_{2}^{\text {cfe }} u_{2}\right)\right\|_{L^{2}\left(\cup \mathcal{T}_{2}^{\Gamma}\right)}^{2} \lesssim\left\|h \nabla^{2} u\right\|_{L^{2}\left(\Omega_{2}\right)}^{2} .
$$

The combination of (3.4), (3.5), (3.6), (3.9), and (3.10) proves the assertion.

3.2. Non-conformity. If the solution is sufficiently smooth, i.e., $u^{\star} \in H^{3 / 2}\left(\Omega_{1} \cup\right.$ $\Omega_{2}$ ), the second term in (3.1) can be estimated using Greens's identity, (2.1), (2.8), the classical jump relation, and the Cauchy-Schwarz inequality as follows:

$$
\sup _{0 \neq v \in S^{\text {cfe }}} \frac{\left|\mathfrak{a}\left(u^{\star}-u^{\text {cfe }}, v\right)\right|}{\|v\|} \leq C\left\|\frac{\partial u^{\star}}{\partial \nu_{\Omega_{1}}}\right\|_{L^{2}(\Gamma)} \sup _{0 \neq v \in S^{\text {cfe }}} \frac{\left\|[[v]]_{\Gamma}\right\|_{L^{2}(\Gamma)}}{\|v\|} .
$$

Here, $\nu_{\Omega_{1}}$ denotes the outer normal of $\Omega_{1}$ and $[[v]]_{\Gamma}$ denotes the jump of $v$ across $\Gamma$. By picking up ideas from [18, Lemma 4.9], one checks that the discontinuity $[[v]]_{\Gamma}$ is in fact small.

Lemma 3.2 (Non-conformity). There is a constant $C=C\left(C_{1}, C_{3}\right)>0$ with $C_{3}:=$ $\max _{T \in \mathcal{T}_{1}^{\Gamma}: T \cap \Gamma \neq \emptyset}|\Gamma \cap T| / \operatorname{diam}(T)^{(d-1)}$, such that

$$
\left\|[[v]]_{\Gamma}\right\|_{L^{2}(\Gamma)} \leq C\|h\|_{L^{\infty}(\Omega)}\left\|h_{1}^{\Gamma} / h^{3 / 2}\right\|_{L^{\infty}\left(\bigcup\left\{T \in \mathcal{T}_{1}^{\Gamma}: T \cap \Gamma \neq \emptyset\right\}\right)}\|\| v\|\|
$$

for all $v \in S^{\mathrm{cfe}}$.

Proof. Let $v=\mathcal{P}^{\text {cfe }}\left(\mathcal{I}_{\mathcal{T}_{1}} v, \mathcal{I}_{\mathcal{T}_{2}} v\right) \in S^{\text {cfe }}$. Let $t \in \mathcal{T}_{1}^{\Gamma}$ with $t \cap \Gamma \neq \emptyset$. We start with some pointwise estimate of the jump of $v$ on $t$ :

$$
\|[[v]]\|_{L^{\infty}(\Gamma \cap t)}=\left\|\left.v\right|_{\Omega_{2}}-\left.v\right|_{\Omega_{1}}\right\|_{L^{\infty}(\Gamma \cap t)} \leq\left\|\left.v\right|_{\Omega_{2}}-\left.v\right|_{\Omega_{1}}\right\|_{L^{\infty}(t)},
$$

where $\left.v\right|_{\Omega_{1}}$ (resp. $\left.u\right|_{\Omega_{2}}$ ) is identified with its unique affine extension onto $t$. The definitions (2.6) and (2.7) yield

$$
\begin{aligned}
\|[[v]]\|_{L^{\infty}(\Gamma \cap t)}=\max _{y \in V(t)}|v|_{\Omega_{2}}(y)-\left.v\right|_{\Omega_{2}}\left(y^{\Gamma}\right) & -\left\langle\nabla \mathcal{I}_{T_{y}^{1}} v, y-y^{\Gamma}\right\rangle \mid \\
& \lesssim \operatorname{diam}(t)\left\|h^{-d / 2} \nabla v\right\|_{L^{2}\left(T_{y}^{1} \cup T_{y}^{2}\right)} .
\end{aligned}
$$


Hence, the $L^{2}$-norm of $v$ on $\partial \Omega$ is estimated as follows:

$$
\begin{aligned}
&\|v\|_{L^{2}(\Gamma)}^{2} \leq \sum_{T_{1} \in \mathcal{T}_{1}, T_{2} \in \mathcal{T}_{2}} \sum_{t \in \mathcal{T}_{1}^{\Gamma}: t \cap \Gamma \neq \emptyset, T_{t}^{k}=T_{k}}|\Gamma \cap t|\|[[v]]\|_{L^{\infty}(t)}^{2} \\
& \qquad \sum_{T_{1} \in \mathcal{T}_{1}, T_{2} \in \mathcal{T}_{2}} \sum_{t \in \mathcal{T}_{1}^{\Gamma}: t \cap \Gamma \neq \emptyset, T_{t}^{k}=T_{k}} \frac{|\Gamma \cap t| \operatorname{diam}(t)^{2}}{\operatorname{diam}(T)^{d}}\|\nabla v\|_{L^{2}\left(T_{1} \cup T_{2}\right)}^{2} \\
& \lesssim\left\|h_{1}^{\Gamma / \sqrt{h}}\right\|_{L^{\infty}\left(\cup\left\{t \in \mathcal{T}_{1}^{\Gamma}: t \cap \Gamma \neq \emptyset\right\}\right)}^{2}\|\nabla v\|_{L^{2}(\Omega)}^{2} .
\end{aligned}
$$

If $\left.h_{1}^{\Gamma}\right|_{\Gamma}$ is chosen proportional to $h^{(3 / 2)}$, as it is assumed in (2.9), Theorem 2.3 follows from (3.1), Lemma 3.1 and Lemma 3.2

Remark 3.3. The constant $C_{3}$ introduced in Lemma 3.2 reflects the smoothness of the interface $\Gamma$. Note that $C_{3}$ may be large if $\Gamma$ is highly oscillating. However, the proof of Lemma 3.2 shows that a possibly large constant can be controlled by simply choosing $\left.h^{\Gamma}\right|_{\Gamma}$ appropriately small. This modification concerns only the submesh $\mathcal{T}_{1}^{\Gamma}$ and does not affect the overall number of degrees of freedom.

\section{Regularity}

This section proves the regularity result (2.11) under the following assumptions on the geometrical setting:

(R1) $\Omega \subset \mathbb{R}^{2}$ is a convex polygon,

(R2) $\Omega_{1}, \Omega_{2} \subset \Omega$ are disjoint open Lipschitz domains with $\bar{\Omega}=\bar{\Omega}_{1} \cup \bar{\Omega}_{2}$, and

(R3) $\Gamma:=\bar{\Omega}_{1} \cap \bar{\Omega}_{2}$ is a $C^{1,1}$ curve that separates $\Omega_{1}$ and $\Omega_{2}$.

The conditions (R1)-(R3) guarantee that both subdomains $\Omega_{k}$ have a piecewise smooth boundary with interior angles less than $\pi$. In particular, the interface is not tangential to $\partial \Omega$ in intersection points $\Gamma \cap \partial \Omega$. Two relevant cases covered by these conditions are depicted in Figure 5.
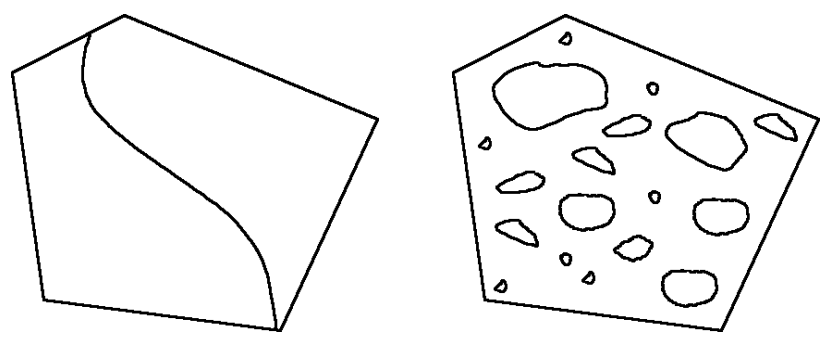

FiguRE 5. Two geometric situations in which conditions (R1)(R3) are satisfied: (left) interface cuts through the boundary of $\Omega$ at some positive angle, (right) smooth separated inclusions dispersed in some matrix.

Under the conditions $(\mathrm{R} 1)-(\mathrm{R} 3)\left[13\right.$. shows piecewise $H^{2}$ regularity in the sense that $f \in L^{2}(\Omega)$ implies $u^{\star} \in H^{2}\left(\Omega_{1} \cup \Omega_{2}\right)$ and

$$
\left\|\nabla^{2} u^{\star}\right\|_{L^{2}\left(\Omega_{1}\right)}+\left\|\nabla^{2} u^{\star}\right\|_{L^{2}\left(\Omega_{2}\right)} \leq C\|f\|_{L^{2}(\Omega)} .
$$


The subsequent theorem clarifies the dependence of the constant in the estimate above on the contrast parameter $a_{\text {cont }}$. In this respect, the theorem generalizes the previous result [4, Theorem B.1], which assumes a smoother interface and, more importantly, the inclusion $\bar{\Omega}_{1} \subset \Omega$ with some positive distance between $\Omega_{1}$ and $\partial \Omega$.

Theorem 4.1. Under the assumptions (R1)-(R3) the unique solution $u^{\star}$ of (2.1) is piecewise smooth, $u^{\star} \in H^{2}\left(\Omega_{1} \cup \Omega_{2}\right)$. Moreover, the estimates (2.11) hold with a generic constant $C_{\text {reg }}$ that does not depend on $f$ and $a_{\text {cont }}$.

Proof. Let $u_{k}^{\star}:=\left.u^{\star}\right|_{\Omega_{k}}$ for $k=1,2$. As discussed earlier, the assumptions (R1)(R3) yield $u_{k}^{\star} \in H^{2}\left(\Omega_{k}\right)$ for $k=1,2$. Since $\Omega_{k}, k=1,2$, is piecewise smooth with interior angles less than $\pi$, classical a priori bounds yield

$$
\begin{aligned}
& \left\|\nabla^{2} u_{1}^{\star}\right\|_{L^{2}\left(\Omega_{1}\right)} \leq C_{\mathrm{reg}}^{\prime}\left(\|f\|_{L^{2}\left(\Omega_{1}\right)}+\left\|u_{1}^{\star}\right\|_{H^{3 / 2}(\Gamma)}\right), \\
& \left\|\nabla^{2} u_{2}^{\star}\right\|_{L^{2}\left(\Omega_{2}\right)} \leq C_{\mathrm{reg}}^{\prime \prime}\left(a_{\mathrm{cont}}^{-1}\|f\|_{L^{2}\left(\Omega_{2}\right)}+\left\|\frac{\partial u_{2}^{\star}}{\partial \nu_{\Omega_{2}}}\right\|_{H^{1 / 2}(\Gamma)}\right) .
\end{aligned}
$$

Since the above estimates are solely performed in the subdomains, the constants $C_{\text {reg }}^{\prime}$ and $C_{\text {reg }}^{\prime \prime}$ do not depend on $a_{\text {cont }}$. The classical jump relations at the interface imply $\left\|u_{1}^{\star}\right\|_{H^{3 / 2}(\Gamma)}=\left\|u_{2}^{\star}\right\|_{H^{3 / 2}(\Gamma)}$ and $\left\|\frac{\partial u_{1}^{\star}}{\partial \nu_{\Omega_{1}}}\right\|_{H^{1 / 2}(\Gamma)}=a_{\text {cont }}\left\|\frac{\partial u_{2}^{\star}}{\partial \nu_{\Omega_{2}}}\right\|_{H^{1 / 2}(\Gamma)}$. Hence,

$$
\begin{aligned}
\left\|\nabla^{2} u_{1}^{\star}\right\|_{L^{2}\left(\Omega_{1}\right)} & \leq C_{\mathrm{reg}}^{\prime}\left(\|f\|_{L^{2}\left(\Omega_{1}\right)}+\left\|u_{2}^{\star}\right\|_{H^{3 / 2}(\Gamma)}\right) \\
\left\|\nabla^{2} u_{2}^{\star}\right\|_{L^{2}\left(\Omega_{2}\right)} & \leq C_{\mathrm{reg}}^{\prime \prime} a_{\text {cont }}^{-1}\left(\|f\|_{L^{2}\left(\Omega_{2}\right)}+\left\|\frac{\partial u_{1}^{\star}}{\partial \nu_{\Omega_{1}}}\right\|_{H^{1 / 2}(\Gamma)}\right) .
\end{aligned}
$$

The combination of (4.2), the trace theorem $\left\|\frac{\partial u_{1}^{\star}}{\partial \nu_{\Omega_{1}}}\right\|_{H^{1 / 2}(\Gamma)} \leq C\left\|u_{1}^{\star}\right\|_{H^{2}\left(\Omega_{1}\right)}$, (14.1), and the trace theorem $\left\|u_{2}^{\star}\right\|_{H^{3 / 2}(\Gamma)} \leq C\left\|u_{2}^{\star}\right\|_{H^{2}\left(\Omega_{2}\right)}$ leads to

$$
\left\|\nabla^{2} u_{2}^{\star}\right\|_{L^{2}\left(\Omega_{2}\right)} \leq C_{\text {reg }}^{\prime \prime \prime} a_{\text {cont }}^{-1}\left(\|f\|_{L^{2}(\Omega)}+\left\|u^{\star}\right\|_{H^{1}(\Omega)}+\left\|\nabla^{2} u_{2}^{\star}\right\|_{L^{2}\left(\Omega_{2}\right)}\right) .
$$

Since $C_{\text {reg }}^{\prime \prime \prime}$ does not depend on $a_{\text {cont }}$, coercivity of the bilinear form $\mathfrak{a}$ and the energy estimate $\left\|\left|u^{\star}\right|\right\| \leq\|f\|_{L^{2}(\Omega)}$ prove the estimate

$$
\left\|\nabla^{2} u_{2}^{\star}\right\|_{L^{2}\left(\Omega_{2}\right)} \leq C_{\text {reg }} a_{\text {cont }}^{-1}\|f\|_{L^{2}(\Omega)},
$$

provided $a_{\text {cont }} \geq C_{\text {reg }}^{\prime \prime \prime} / 2$. Since for small contrast $a_{\text {cont }}<C_{\text {reg }}^{\prime \prime \prime} / 2$ nothing is to show, one assertion is proved. The estimate for $\left\|\nabla^{2} u_{2}^{\star}\right\|_{L^{2}\left(\Omega_{2}\right)}$ is analogous by interchanging the application of (4.2) and (4.1) as well as the corresponding trace inequalities.

For a characterization of the singularities that may appear if the conditions (R1)(R3) are not satisfied, we refer the reader to [2, 6, 10, 11] among many others. A comprehensive regularity analysis for the three-dimensional case is more technical and beyond the scope of this paper; we refer to [12] for necessary conditions under which $H^{2}\left(\Omega_{1} \cup \Omega_{2}\right)$-regularity is achieved. If the geometric setting allows $H^{2}\left(\Omega_{1} \cup\right.$ $\Omega_{2}$ )-regularity, then the proof of (2.11) could be treated in a similar way as in Theorem 4.1

We shall emphasize that the above result is not explicit with respect to the geometric setting, e.g., the constants $C_{\text {reg }}^{\prime}$ and $C_{\text {reg }}^{\prime \prime}$ may depend on oscillations of the interface, minimal distances between inclusions, the distance between inclusions 
and the boundary, etc. The dependence on the geometry is involved and has been studied only for special cases, e.g., the case of densely packed, perfectly conducting, circular inclusions in $2 d$ [17]. We further mention that reularity estimates for the case of diffusive interfaces may be found in [20].

\section{Conclusion}

We have described a finite element method for the Poisson equation with discontinuous diffusion coefficient across some interface. The method does not require the underlying finite element mesh to resolve the interface exactly. Overlapping, and possibly structured, simplicial meshes can be used instead. Moreover, the definition of the basis functions is explicit, and no local problems have to be solved. On a quasi-uniform coarse grid of width $h$, the complexity of our method is proportional to $h^{-d}$, whereas the error is proportional to $h$. This is optimal in comparison with the approximation of a Poisson problem with overall constant coefficient on the same mesh.

This paper focuses on the difficulty of treating discontinuous coefficients. To keep notation and technicalities at a minimum, the simplest possible setting has been chosen. Generalizations, not only to general linear elliptic problems but also saddle point problems such as Stokes' problem, are straightforward with regard to the previous work [18, 19.

\section{REFERENCES}

[1] Roland Becker, Erik Burman, and Peter Hansbo, A Nitsche extended finite element method for incompressible elasticity with discontinuous modulus of elasticity, Comput. Methods Appl. Mech. Engrg. 198 (2009), no. 41-44, 3352-3360, DOI 10.1016/j.cma.2009.06.017. MR2571349 (2011b:74023)

[2] Matthias Blumenfeld, The regularity of interface-problems on corner-regions (Oberwolfach, 1983), Lecture Notes in Math., vol. 1121, Springer, Berlin, 1985, pp. 38-54, DOI 10.1007/BFb0076261. MR806385(87a:35063)

[3] Susanne C. Brenner and L. Ridgway Scott, The mathematical theory of finite element methods, 3rd ed., Texts in Applied Mathematics, vol. 15, Springer, New York, 2008. MR2373954 (2008m:65001)

[4] C.-C. Chu, I. G. Graham, and T.-Y. Hou, A new multiscale finite element method for high-contrast elliptic interface problems, Math. Comp. 79 (2010), no. 272, 1915-1955, DOI 10.1090/S0025-5718-2010-02372-5. MR2684351 (2011j:65267)

[5] Philippe G. Ciarlet, The finite element method for elliptic problems, North-Holland Publishing Co., Amsterdam, 1978. Studies in Mathematics and its Applications, Vol. 4. MR0520174 (58 \#25001)

[6] Monique Dauge and Serge Nicaise, Oblique derivative and interface problems on polygonal domains and networks, Comm. Partial Differential Equations 14 (1989), no. 8-9, 1147-1192, DOI 10.1080/03605308908820649. MR1017069(91a:35046)

[7] W. Hackbusch and S. A. Sauter, Composite finite elements for problems containing small geometric details. Part II: Implementation and numerical results, Computing and Visualization in Science 1 (1997), 15-25.

[8] W. Hackbusch and S. A. Sauter, Composite finite elements for the approximation of PDEs on domains with complicated micro-structures, Numer. Math. 75 (1997), no. 4, 447-472, DOI 10.1007/s002110050248. MR 1431211 (97k:65251)

[9] Anita Hansbo and Peter Hansbo, An unfitted finite element method, based on Nitsche's method, for elliptic interface problems, Comput. Methods Appl. Mech. Engrg. 191 (2002), no. 47-48, 5537-5552, DOI 10.1016/S0045-7825(02)00524-8. MR1941489(2003i:65105)

[10] R. B. Kellogg, Singularities in interface problems, In Numerical Solution of Partial Differential Equations, II (SYNSPADE 1970) (Proc. Sympos., Univ. of Maryland, College Park, Md., 1970), Academic Press, New York, 1971, pp. 351-400. MR0289923 (44 \#7108) 
[11] D. Leguillon and E. Sánchez-Palencia, Computation of singular solutions in elliptic problems and elasticity, John Wiley \& Sons Ltd., Chichester, 1987. MR995254 (90m:73015)

[12] K. Lemrabet, An interface problem in a domain of $\mathbf{R}^{3}$, J. Math. Anal. Appl. 63 (1978), no. 3, 549-562, DOI 10.1016/0022-247X(78)90059-8. MR.493687 (81c:35036)

[13] Keddour Lemrabet, Régularité de la solution d'un problème de transmission (French), J. Math. Pures Appl. (9) 56 (1977), no. 1, 1-38. MR0509312 (58 \#23016)

[14] Jingzhi Li, Jens Markus Melenk, Barbara Wohlmuth, and Jun Zou, Optimal a priori estimates for higher order finite elements for elliptic interface problems, Appl. Numer. Math. 60 (2010), no. 1-2, 19-37, DOI 10.1016/j.apnum.2009.08.005. MR2566075 (2011a:65370)

[15] M. Ohlberger and M. Rumpf, Hierarchical and adaptive visualization on nested grids, Computing 59 (1997), no. 4, 365-385, DOI 10.1007/BF02684418. MR1486386 (98h:65058)

[16] D. Peterseim, The composite mini element: A mixed FEM for the Stokes equations on complicated domains, Ph.D. thesis, Universität Zürich, http://www.dissertationen.uzh.ch/, 2007.

[17] Daniel Peterseim, Robustness of finite element simulations in densely packed random particle composites, Netw. Heterog. Media 7 (2012), no. 1, 113-126, DOI 10.3934/nhm.2012.7.113. MR2908612

[18] Daniel Peterseim and Stefan A. Sauter, The composite mini element-coarse mesh computation of Stokes flows on complicated domains, SIAM J. Numer. Anal. 46 (2008), no. 6, 3181-3206, DOI 10.1137/070704356. MR2439507(2009e:76107)

[19] Daniel Peterseim and Stefan A. Sauter, Finite element methods for the Stokes problem on complicated domains, Comput. Methods Appl. Mech. Engrg. 200 (2011), no. 33-36, 26112623, DOI 10.1016/j.cma.2011.04.017. MR2812028(2012e:76074)

[20] D. Peterseim and S. Sauter, Finite Elements for Elliptic Problems with Highly Varying, Nonperiodic Diffusion Matrix, Multiscale Model. Simul. 10 (2012), no. 3, 665-695, DOI 10.1137/10081839X. MR3022017

[21] M. Rech, Composite finite elements: An adaptive two-scale approach to the non-conforming approximation of Dirichlet problems on complicated domains, Ph.D. thesis, Universität Zürich, 2006.

[22] M. Rech, S. Sauter, and A. Smolianski, Two-scale composite finite element method for Dirichlet problems on complicated domains, Numer. Math. 102 (2006), no. 4, 681-708, DOI 10.1007/s00211-005-0654-x. MR2207285 (2006m:65283)

[23] S. A. Sauter and R. Warnke, Extension operators and approximation on domains containing small geometric details, East-West J. Numer. Math. 7 (1999), no. 1, 61-77. MR.1683936 (2000c:65105)

[24] S. A. Sauter and R. Warnke, Composite finite elements for elliptic boundary value problems with discontinuous coefficients, Computing 77 (2006), no. 1, 29-55, DOI 10.1007/s00607-0050150-2. MR2207954 (2006k:65335)

[25] Elias M. Stein, Singular integrals and differentiability properties of functions, Princeton Mathematical Series, No. 30, Princeton University Press, Princeton, N.J., 1970. MR0290095 (44 \#7280)

Institut für Mathematik, Humboldt-Universität Zu Berlin, Unter den Linden 6, 10099 Berlin, Germany

Current address: Institut für Numerische Simulation der Universität Bonn, Wegelerstr. 6, 53115 Bonn, Germany

E-mail address: peterseim@ins.uni-bonn.de 